Received: 09.10.2021

Revised: 09.12.2021

Accepted: 24.12 .2021

DOI: $10.17804 / 2410-9908.2021 .6 .045-053$

\title{
DETERMINATION OF THE SURFACE OF DEFECT LOCATION AND RESTORATION OF ITS GEOMETRIC PARAMETERS BY THE MFL METHOD WITH ONE-SIDED ACCESS TO THE FERROMAGNETIC PLATE
}

\author{
A. V. Nikitin ${ }^{\text {a)* }}$, A. V. Mikhaylov ${ }^{\text {b) }}$, Yu. L. Gobov ${ }^{\text {() }}$, and S. E. Popov ${ }^{\text {d) }}$ \\ M.N. Miheev Institute of Metal Physics, Ural Branch of the Russian Academy of Sciences, \\ 18 S. Kovalevskoy St., Ekaterinburg, 620108, Russian Federation \\ a) (D) https://orcid.org/0000-0002-4448-9835 an@imp.uran.ru; \\ b) (D https://orcid.org/0000-0001-9342-8986 @ikhaylov@imp.uran.ru; \\ c) (D) https://orcid.org/0000-0003-3986-2479 go@imp.uran.ru; \\ d) (ID https://orcid.org/0000-0002-0041-8760 @ sergeyeduardovichpopov@ gmail.com \\ *Corresponding author: E-mail: an@imp.uran.ru \\ Address for correspondence: ul. S. Kovalevskoy, 18, Ekaterinburg, 620108, Russian Federation \\ Tel.: +7 (343) 3783885
}

The paper presents a theoretical and experimental confirmation of the technique allowing the surface of defect location and its geometric parameters to be determined from measuring the values of the magnetic field components above one of the surfaces of a magnetically soft ferromagnetic plate. The technique allows one to take into account the nonlinearity of the response of a ferromagnet to an external magnetostatic field.

Keywords: magnetically soft ferromagnet, metal surface discontinuity defect, polar and tangential magnetization, MFL method, scalar potential of magnetostatic field, equipotential surface, magnetic field line, inverse geometric problem of magnetostatics, defect characterization.

\section{Acknowledgment}

The work was carried out under the state assignment on the topic of Diagnostics, No. AAAAA18-118020690196-3.

\section{References}

1. Available at: https://pipelineoperators.org/

2. Available at: https://ntengd.com/uslugi/article_post/vnutritrubnaya-diagnostika-spomoshchyumagnitnykh-defektoskopov-vysokogo-razresheniya

3. Bolshakova V.V., Kukin N.A., Dymkin G.Ya. On possibility of NDT magnetic methods for evaluation of strain-stress state of pipelines. Izvestiya peterburgskogo universiteta putey soobshcheniya, 2014, No. 4 (41), pp. 92-98. (In Russian).

4. Slesarev D.A., Abakumov A.A. Data processing and representation in the MFL method for nondestructive testing. Russian Journal of Nondestructive Testing, 2013, vol. 49 (9), pp. 493-498. DOI: $10.1134 / \mathrm{S} 106183091309009 \mathrm{X}$.

5. Kanaykin V.A. Vnutritrubnaya magnitnaya defektoskopiya magistralnykh truboprovodov [In-line magnetic flaw detection of main pipelines, ed. by A.F. Matvienko]. Ekaterinburg, UrO RAN Publ., 2009, 307 p. 
6. Dyakin V.V., Kudryashova O.V., Raevskii V.Y. Stray field of plate with a surface defect in a homogeneous external field. Russian Journal of Nondestructive Testing, 2018, vol. 54, No. 12, pp. 840-848. DOI: 10.1134/S1061830918120033.

7. Gobov Yu.L., Nikitin A.V., Popov S.E. Solving the Inverse Geometric Problem of Magnetostatics for Corrosion Defects. Russian Journal of nondestructive testing, 2018, vol. 54, pp. 726-732. DOI: 10.1134/S1061830918100042.

8. Gobov Yu.L., Nikitin A.V., Popov S.E. Solving the Inverse Geometric Problem of Magnetostatics for Corrosion Defects with Allowance for Nonlinear Properties of Ferromagnet. Russian Journal of nondestructive testing, 2018, vol. 54, pp. 849 - 854. DOI: 10.1134/S1061830918120045.

9. Nikitin A.V., Mikhaylov A.V., Petrov A.S., Popov S.E., Gobov Yu.L. A technique for practical reconstruction of the shape parameters of two-dimensional surface defects taking into account the nonlinear properties of a ferromagnet. Defektoskopiya, 2021, vol. 12, pp. 46-55. DOI: $10.31857 / \mathrm{S} 0130308221120058$.

10. Gobov Yu.L., Popov S.E. Reconstructing the topography of surface defects of ferromagnets in a normal magnetization field. Russian journal of nondestructive testing, 2021, vol. 57, pp. 303-309. DOI: $10.1134 / \mathrm{S} 1061830921040057$.

11. Jackson J.D. Klassicheskaya elektrodinamika [Jackson John David. Classical electrodynamics, New York-London : John Wiley\&Sons, Inc., 1962], Rus. transl., Moscow, Mir Publ., 1965, 702 p. 
Подана в журнал: 09.10.2021

УДК 620.179.14

DOI: $10.17804 / 2410-9908.2021 .6 .045-053$

\title{
ОПРЕДЕЛЕНИЕ ЛОКАЛИЗАЦИИ ДЕФЕКТА, А ТАКЖЕ ВОССТАНОВЛЕНИЕ МЕТОДОМ МЕL ЕГО ГЕОМЕТРИЧЕСКИХ ПАРАМЕТРОВ ПРИ ОДНОСТОРОННЕМ ДОСТУПЕ К ФЕРРОМАГНИТНОЙ ПЛАСТИНЕ
}

\author{
А. В. Никитин ${ }^{\text {a) }}$, А. В. Михайлов ${ }^{\text {б) }}$, Ю. Л. Гобов ${ }^{\text {в) }}$, С. Э. Попов ${ }^{\text {г) }}$ \\ Федеральное государственное бюджетное учреждение науки \\ Институт физики металлов им. М.Н. Михеева Уральского отделения Российской академии наук, \\ д. 18, ул. С. Ковалевской, Екатеринбург, 620108, Российская Федерация \\ a) (D) https://orcid.org/0000-0002-4448-9835 @ an@imp.uran.ru; \\ б) (D https://orcid.org/0000-0001-9342-8986 @ikhaylov@imp.uran.ru; \\ в) (D) https://orcid.org/0000-0003-3986-2479 oimp.uran.ru; \\ г) (D) https://orcid.org/0000-0002-0041-8760 @ sergeyeduardovichpopov@ gmail.com \\ *Ответственный автор. Электронная почта: an@imp.uran.ru \\ Адрес для переписки: Российская Федерация, 620108, г. Екатеринбург, ул. С. Ковалевской, 18 \\ Тел.: +7 (343)378-38-85
}

Представлено теоретическое и экспериментальное подтверждение методики позволяющей определять поверхность расположения дефекта и его геометрические параметры по результатам измерений величин компонент магнитного поля над одной из поверхностей магнитомягкой ферромагнитной пластины. Методика учитывает нелинейность отклика ферромагнетика на внешнее магнитостатическое поле.

Ключевые слова: магнитомягкий ферромагнетик, поверхностный дефект потери сплошности металла, полюсное и тангенциальное намагничивание, метод MFL, скалярный потенциал магнитостатического поля, эквипотенциальная поверхность, силовая магнитная линия, обратная геометрическая задача магнитостатики, дефектометрия.

\section{1. Введение}

Настоящая работа посвящена определению геометрических параметров поверхностных дефектов потери сплошности металла (магнитомягкого ферромагнетика) методом MFL в тангенциальном намагничивающем поле, а также в нормальном к поверхности пластины магнитном поле. В работе не рассматриваются трещины и трещиноподобные дефекты (определения дефектов можно найти в [1]).

Стали, представляющие собой магнитомягкие ферромагнетики, используются во многих отраслях промышленности и на транспорте. В частности, трубы магистральных трубопроводов, бурильные и насосно-компрессорные трубы изготавливают из магнитомягких ферромагнетиков.

Широкое распространение метода MFL (стандартно используется тангенциальное намагничивание) для диагностики пластин из ферромагнитных материалов обусловлено следующими факторами: портативностью первичных преобразователей, отсутствием особых требований к подготовке поверхности металла, высокой скоростью сканирования, простотой автоматизации измерений [2-4]. При этом во многих отраслях индустрии и на транспорте типичной является ситуация, когда магнитное поле удается измерить лишь на одной из поверхностей прямой или изогнутой металлической пластины. В этой ситуации при помощи только метода MFL невозможно указать, на какой поверхности расположен дефект. Вместе с 
тем в настоящее время метод MFL является индикаторным методом и, если требуется более точное определение геометрических параметров дефектов, то как правило проводят дополнительную обработку полученных методом MFL магнитограмм; сигналы от дефектов на магнитограмме сравнивают с сигналами эталонных дефектов [5-6].

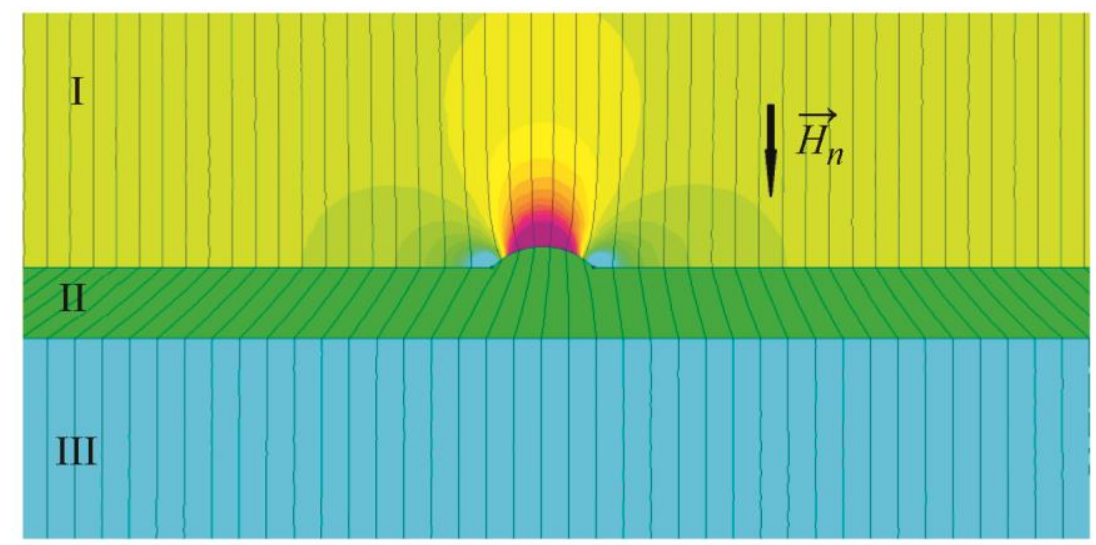

Рис. 1. Распределение силовых линий статического магнитного поля вблизи дефекта типа «наплавка» при нормальном намагничивании. $I$ - область воздуха между системой намагничивания и пластиной, в этой области расположены магнитные преобразователи; II - металлическая пластина; III - область воздуха за металлической пластиной.

$$
\overrightarrow{H_{n}}-\text { поле намагничивания }
$$

Реализация методики, в которой определяются поверхность расположения дефекта и его геометрические параметры, позволит осуществлять дефектометрию поверхностных дефектов потери сплошности металла в автоматическом режиме, что станет базой для создания использующих MFL метод приборов нового поколения.

В этих целях были проведены численное и экспериментальное исследования по возможности определения геометрических параметров поверхностных дефектов MFL методом как при стандартном тангенциальном намагничивании пластины [7-9], так и при намагничивании пластины нормальным к ее поверхности магнитным полем [10].

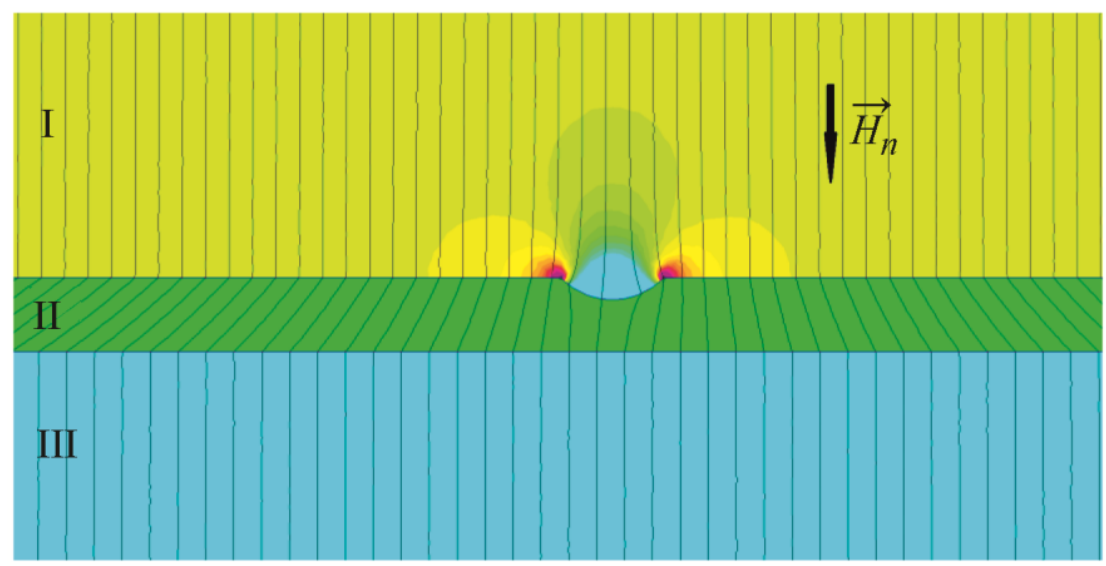

Рис. 2. Распределение силовых линий статического магнитного поля вблизи дефекта потери металла при нормальном намагничивании: $I$ - область воздуха между системой намагничивания и пластиной, в этой области расположены магнитные преобразователи; II - металлическая пластина; III - область воздуха за металлической пластиной;

$$
\overrightarrow{H_{n}} \text { - поле намагничивания }
$$


На рис. 1-4 показаны результаты решения прямой задачи магнитостатики, полученные в пакете FEMM [10]. На всех рисунках подразумевается, что магнитные преобразователи находятся в области $I$, на расстоянии 2 мм от бездефектной поверхности ферромагнетика. Область металлической пластины (обозначена на рисунках римской цифрой II) содержит как дефекты потери металла, так и дефекты типа «наплавка». Из рисунков следует, что при нормальном намагничивании пластины магнитные преобразователи принимают сигналы только от дефектов, расположенных на ближайшей к преобразователям поверхности пластины (рис. 1-3). Сигналы от дефектов с противоположной поверхности экранируются магнитомягким материалом. При тангенциальном намагничивании пластины магнитные преобразователи получают сигналы от дефектов, расположенных на обеих поверхностях пластины (рис. 4). Это позволяет определить поверхность, на которой расположен тот или иной дефект.

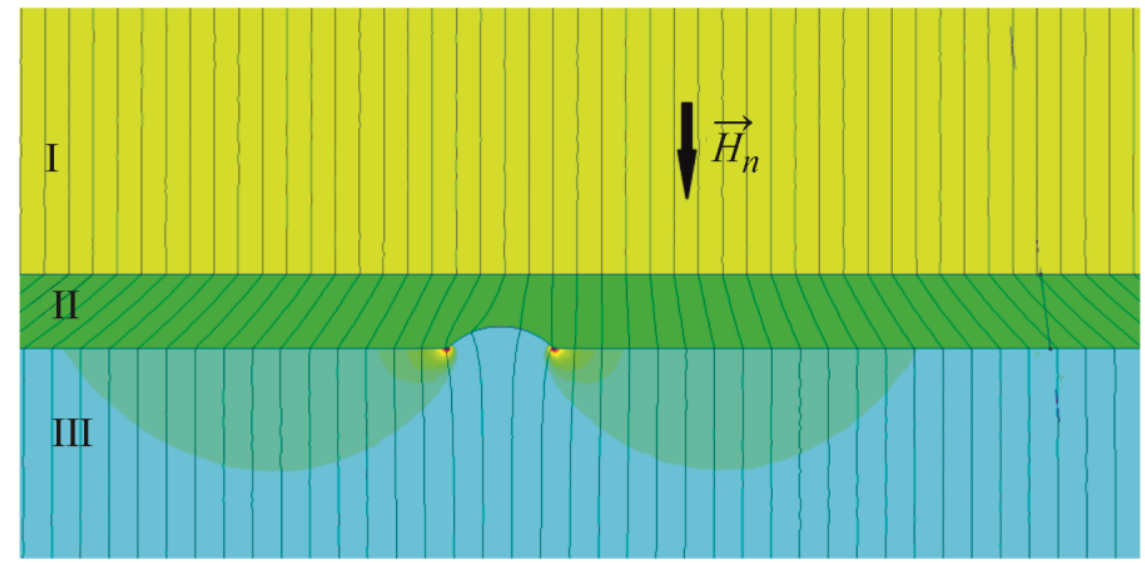

Рис. 3. Распределение силовых линий статического магнитного поля вблизи дефекта потери металла, расположенного на противоположной к магнитным преобразователям поверхности пластины, при нормальном намагничивании: $I$ - область воздуха между системой намагничивания и пластиной, в этой области расположены магнитные преобразователи; II - металлическая пластина; III - область воздуха за металлической пластиной; $\overrightarrow{H_{n}}$ - поле намагничивания

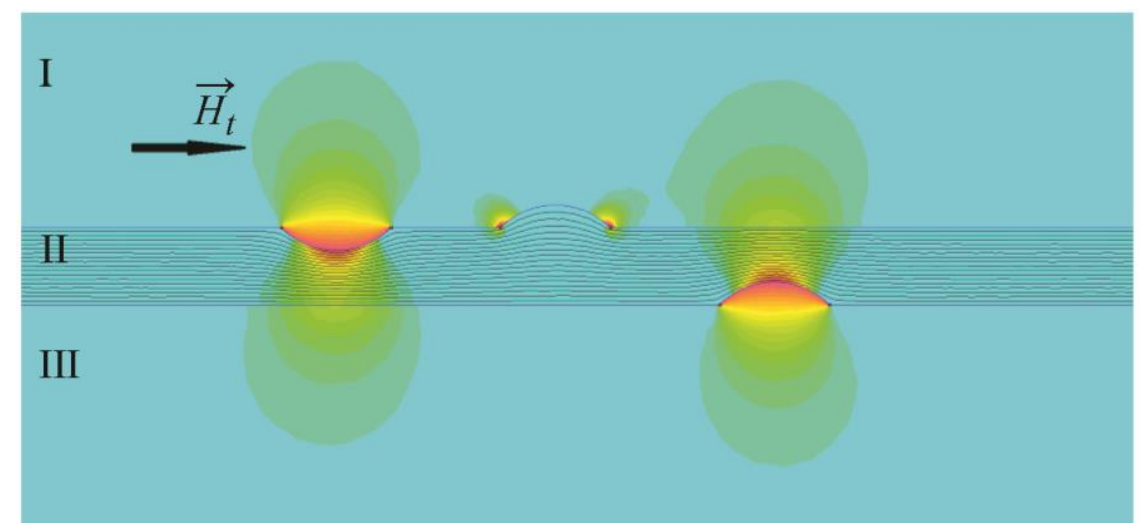

Рис. 4. Силовые линии и изолинии магнитного поля рассеяния дефектов при тангенциальном намагничивании: $I$ - область воздуха между системой намагничивания и пластиной, в этой области расположены магнитные преобразователи; II - металлическая пластина; III - область воздуха за металлической пластиной; $\overrightarrow{H_{t}}$ - поле намагничивания 


\section{2. Определение геометрических параметров поверхностных дефектов методом MFL в тангенциальном стационарном намагничивающем поле}

В случае тангенциального намагничивания определим геометрические параметры дефектов, расположенных на противоположной от магнитных преобразователей поверхности пластины (рис. 4, дефект справа на нижней поверхности металла).

Для приближенного решения обратной геометрической задачи магнитостатики воспользуемся тем фактом, что выход магнитного потока из металла в магнитомягких ферромагнетиках даже в области дефекта составляет всего лишь несколько процентов, которым можно пренебречь. Далее, восстановив поле в металле от верхней поверхности к нижней и даже ниже, считая, что ниже всюду находится металл, можно построить 2 силовые линии (в двухмерном случае): одну на уровне верхней бездефектной границы пластины, другую - на уровне нижней границы пластины, содержащей дефект начиная с бездефектной области. Выход магнитного потока между этими двумя линиями невозможен (силовые линии не пересекаются), кроме того, форма силовой магнитной линии на нижней поверхности пластины соответствует измеренному в области I магнитному полю рассеяния дефекта. Таким образом, можно с хорошей точностью определить глубину дефекта. Раскрытие дефекта удобней оценивать по топографии магнитного поля, например на границе металл-воздух со стороны металла.

Для восстановления поля в металле используем следующую методику. По измеренным компонентам поля в области I (вблизи границы металл-воздух со стороны воздуха), используя выражения сопряжения на границе двух сред, можно пересчитать компоненты напряженности магнитного поля и магнитной индукции на границу металл-воздух со стороны металла. Эти компоненты магнитного поля, а также их производные по оси абсцисс будут начальными условиями для дальнейшей процедуры.

С учетом нелинейной зависимости $\vec{B}(|\vec{H}|)$ запишем уравнения Максвелла для ферромагнитной среды - в случае магнитостатики:

$$
\operatorname{div} \vec{B}=0 ; \operatorname{rot} \vec{H}=0 ; \vec{B}=\mu(|\vec{H}|) * \vec{H},
$$

где $\vec{B}$ и $\vec{H}$ - векторы магнитной индукции и напряженности магнитного поля соответственно, a $|\vec{H}|=\sqrt{\left(H_{x}\right)^{2}+\left(H_{z}\right)^{2}}$.

В качестве зависимости $\mu(H)$ возьмем известную зависимость $\mu(H)$ для стали 20.

Перепишем систему уравнений (1) в форме конечных разностей - в формулировке задачи Коши (2). Начальные условия для задачи Коши на уровне $Z_{0}$ (значения $B_{z}\left(x, Z_{0}\right), H_{x}\left(x, Z_{0}\right), \frac{\Delta_{z} * d B_{x}}{d x}$, $\left.\frac{\Delta_{z} * d H_{z}}{d x}\right)$ были определены ранее.

$$
\begin{gathered}
B_{z}\left(x, Z_{0}-\Delta_{z}\right)=B_{z}\left(x, Z_{0}\right)+\frac{\Delta_{z} * d B_{x}}{d x} \\
H_{x}\left(x, Z_{0}-\Delta_{z}\right)=H_{x}\left(x, Z_{0}\right)-\frac{\Delta_{z} * d H_{z}}{d x} \\
B_{z}\left(x, Z_{0}-\Delta z\right)=\mu\left(\sqrt{H_{x}\left(x, Z_{0}-\Delta_{z}\right)^{2}+H_{z}\left(x, Z_{0}-\Delta_{z}\right)^{2}}\right) * H_{z}\left(x, Z_{0}-\Delta_{z}\right) \\
B_{x}\left(x, Z_{0}-\Delta z\right)=\mu\left(\sqrt{H_{x}\left(x, Z_{0}-\Delta_{z}\right)^{2}+H_{z}\left(x, Z_{0}-\Delta_{z}\right)^{2}}\right) * H_{x}\left(x, Z_{0}-\Delta_{z}\right),
\end{gathered}
$$

где $Z_{0}$ - указывает уровень границы раздела металл-воздух со стороны металла; $\Delta_{z}$ - шаг дискретизации по оси OZ при пересчете поля с верхнего слоя на нижний слой. 
Теперь, решая задачу Коши (2), мы можем восстановить магнитное поле от уровня границы металл-воздух со стороны металла, вниз до нижней поверхности пластины и даже ниже, всюду считая, что ниже находится металл. Далее по восстановленным значениям магнитного поля в металле вычисляются координаты описанных выше силовых линий.

На рис. 5 приведена силовая линия, полученная в результате проведения реального эксперимента и с хорошей точностью описывающая глубину дефекта [9].

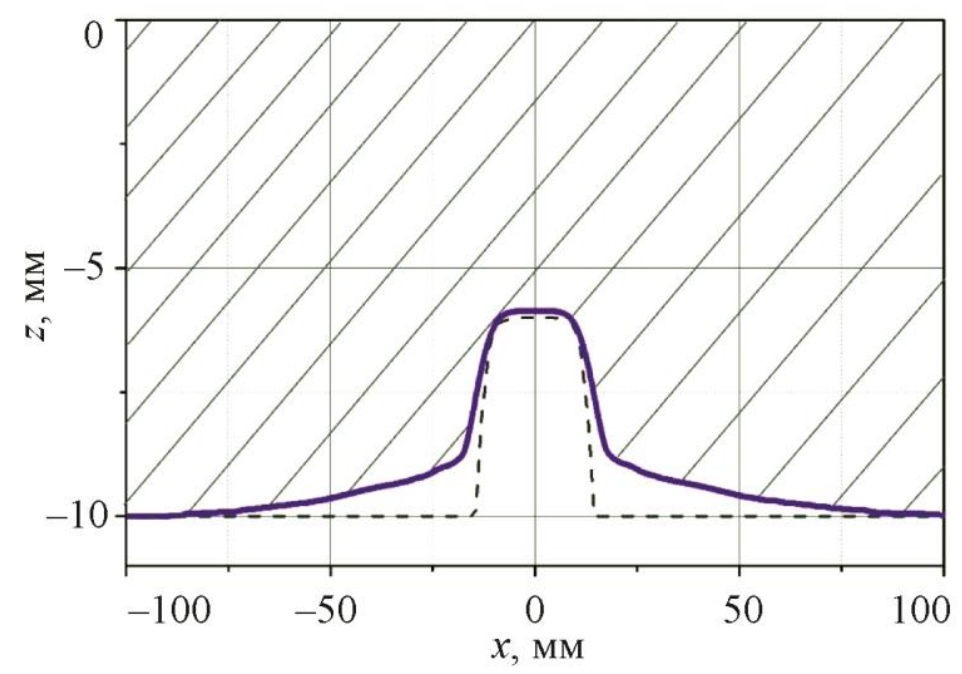

Рис. 5. Сечение восстановленной формы дефекта. Толщина пластины 10 мм, глубина дефекта 4 мм, раскрытие дефекта 29 мм. Компоненты магнитного поля были получены экспериментально, над бездефектной поверхностью металла (сталь 20). Пунктирная линия показывает реальную форму дефекта; сплошная линия представляет вычисленную силовую магнитную линию

\section{3. Определение геометрических параметров поверхностных дефектов методом MFL в нормальном стационарном намагничивающем поле}

В случае нормального намагничивания будем определять геометрические параметры дефектов на ближней к магнитным преобразователям поверхности пластины (рис. 1 и 2).

Для определения геометрических параметров дефектов необходимо намагничивать исследуемый объект до величин 0,3-0,8 Тл. При таком намагничивающем поле поверхность исследуемого материала не намагничивается до насыщения и материал остается магнитомягким с магнитной проницаемостью $\mu \sim 1000$. Такое большое значение магнитной проницаемости позволяет воспользоваться результатами, полученными для скалярного потенциала в электростатике [11], и считать поверхность металла эквипотенциальной поверхностью также и для магнитностатического скалярного потенциала.

Для рассчета геометрической формы дефекта предположим, что нормальная составляющая магнитного поля вблизи дефекта изменяется линейным образом по оси OZ (нормаль к поверхности).

Используя измеренные компоненты магнитного поля на уровне системы преобразователей в области $I$, вычислим значения скалярного магнитостатического потенциала на этом уровне. Далее, используя ряд Тейлора и учитывая линейность по $\mathrm{z}$ магнитного поля $H_{z}$ вблизи дефекта, получим приближенное выражение для значения скалярного потенциала на поверхности дефекта. Итак, 


$$
\begin{aligned}
\varphi(x, y, z)=\varphi\left(x, y, z_{0}\right)- & H_{z}\left(x, y, z_{0}\right) *\left(z(x, y)-z_{0}(x, y)\right)-\frac{1}{2} H_{z z}\left(x, y, z_{0}\right) \times \\
& \times\left(z(x, y)-z_{0}(x, y)\right)^{2},
\end{aligned}
$$

где $z_{0}(x, y)$ - это уровень измерений магнитного поля; $z(x, y)$ - поверхность дефекта. $H_{z}\left(x, y, z_{0}\right)$ это нормальная составляющая магнитного поля на уровне измерений; $H_{z z}\left(x, y, z_{0}\right)$ - производная нормальной составляющей магнитного поля по оси $\mathrm{OZ}$.

Так как поверхность дефекта является эквипотенциальной поверхностью, положив $\varphi(x, y, z)=0$, из выражения для значения скалярного потенциала находим $z(x, y)$.

На рис. 6 и 7 представлены сечения поверхностных дефектов, полученные в результате проведения реального эксперимента [10].

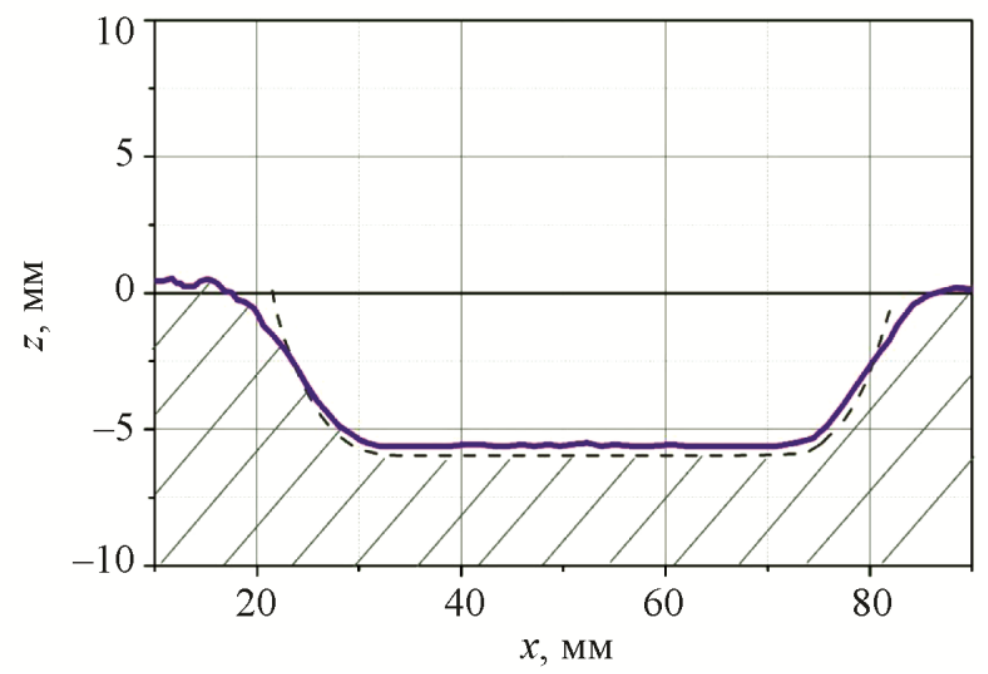

Рис. 6. Сечение восстановленной топографии дефекта потери металла.

Сплошная линия - результат восстановления, пунктирная линия - сечение профиля дефекта, использовавшегося при расчетах методом конечных элементов

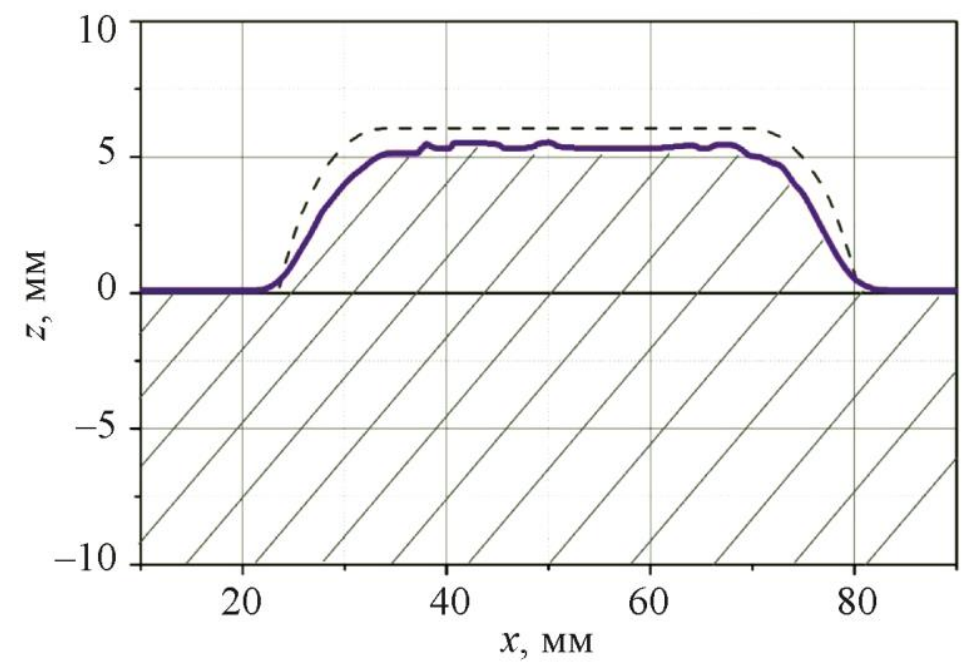

Рис. 7. Сечение восстановленной топографии дефекта типа «наплавка».

Сплошная линия - результат восстановления, пунктирная линия - сечение профиля дефекта, использовавшегося при расчетах методом конечных элементов 


\section{4. Выводы}

Дополнение метода MFL в тангенциальном поле методом MFL в нормальном поле намагничивания позволяет определять поверхность нахождения дефектов. По полученным в результате применения этих методов магнитограммам можно определять геометрические параметры поверхностных дефектов.

\section{Благодарности}

Работа выполнена в рамках государственного задания по теме "Диагностика», № AAAA-A18-118020690196-3.

\section{Литература}

1. URL: https://pipelineoperators.org/

2. URL: https://ntcngd.com/uslugi/article_post/vnutritrubnaya-diagnostika-spomoshchyumagnitnykh-defektoskopov-vysokogo-razresheniya

3. Большакова В. В., Кукин Н. А., Дымкин Г. Я. О возможности применения магнитных методов неразрушающего контроля для оценки напряженно-деформированного состояния трубопроводов // Известия Петербургского университета путей сообщения. - 2014. No. 4 (41). - P. 92-98.

4. Slesarev D. A., Abakumov A. A. Data processing and representation in the MFL method for nondestructive testing // Russian Journal of Nondestructive Testing. - 2013. - Vol. 49 (9). P. 493-498. - DOI: 10.1134/S106183091309009X.

5. Канайкин В. А. Внутритрубная магнитная дефектоскопия магистральных трубопроводов / под ред. А. Ф. Матвиенко. - Екатеринбург : УрО РАН, 2009. - 307 с.

6. Dyakin V. V., Kudryashova O. V., Raevskii V. Y. Stray field of plate with a surface defect in a homogeneous external field // Russian Journal of Nondestructive Testing. - 2018. - Vol. 54, No. 12. - P. 840-848. - DOI: 10.1134/S1061830918120033.

7. Gobov Yu. L., Nikitin A. V., Popov S. E. Solving the Inverse Geometric Problem of Magnetostatics for Corrosion Defects // Russian Journal of Nondestructive Testing. - 2018. Vol. 54. - P. 726-732. - DOI: 10.1134/S1061830918100042.

8. Gobov Yu. L., Nikitin A. V., Popov S. E. Solving the Inverse Geometric Problem of Magnetostatics for Corrosion Defects with Allowance for Nonlinear Properties of Ferromagnet // Russian Journal of nondestructive testing. - 2018. - Vol. 54. - P. 849-854. - DOI: 10.1134/S1061830918120045.

9. Методика практического восстановления параметров формы поверхностных двухмерных дефектов с учетом нелинейных свойств ферромагнетика / А. В. Никитин, А. В. Михайлов, А. С. Петров, С. Э. Попов, Ю. Л. Гобов // Дефектоскопия. - 2021. - Vol. 12. C. $46-55$.

10. Gobov Yu. L., Popov S. E. Reconstructing the topography of surface defects of ferromagnets in a normal magnetization field // Russian Journal of nondestructive testing. 2021. - Vol. 57. - P. 303-309. - DOI: 10.1134/S1061830921040057.

11. Дж. Д. Джексон. Классическая электродинамика / пер. с англ. - М. : Мир, 1965 - 702 с. 